\title{
Grenzen und Sprachgrenzen in der Sprachwissenschaft
}

\author{
Marek Nekula
}

\begin{abstract}
Der Beitrag befasst sich mit der Konzeptualisierung der Grenze in der Sprachwissenschaft, und zwar in der Dialektologie, Variationslinguistik und Interlinguistik, die sich mit Interaktion und Abgrenzung von Varietäten einer Sprache bzw. von zwei oder mehreren Sprachen auseinandersetzen. In Bezug auf die Sprache im sozialen Raum wird dargestellt, wie die Herausbildung (und Aufrechterhaltung) der intralingualen Sprachgrenze zwischen zwei Varietäten einer Sprache und der interlingualen Sprachgrenze zwischen zwei Sprachen in Bezug auf die physisch erlebten und/oder mental vorgestellten horizontalen (territorialen) und vertikalen (sozialen) Grenzen in der Sprachwissenschaft interpretiert wird.
\end{abstract}

\section{Schlagwörter}

Hybridwörter, intralinguale/interlinguale Grenzen, Überschreitung von Sprachgrenzen, Codeswitching/Codemixing

\section{Grenzen in der Sprachwissenschaft: eine Einleitung}

Auch wenn die Herangehensweise der Linguistik an das Verhältnis zwischen Sprache/n und Grenze nach Peter Auer (2005, S. 150) stärker empirisch als theoretisch bestimmt sei (mehr zu Sprache und Raum vgl. auch Auer/Schmidt 2010), dürfte der Umgang linguistischer Disziplinen mit Grenzen bzw. Sprachgrenzen für den vorliegenden Band dennoch von Interesse sein. Daher geht man in diesem Beitrag anhand von deutschen, tschechischen, deutsch-tschechischen und anderen Beispielen nicht nur auf die Ausprägung und den Wandel von Sprachgrenzen, sondern auch auf deren sprachwissenschaftliche Konzeptualisierung ein.

Den Grenzen bzw. Sprachgrenzen und deren Überschreitung, die für diesen Band von Interesse sind, kann man in interdisziplinären Zugängen begegnen, die Sprache und Raum verknüpfen. Gemeint sind Dialektologie, Areallinguistik, Geolinguistik, Soziolinguistik, Interlinguistik, aber auch Sprachplanungs- bzw. Sprachmanagementtheorie. Diese setzen Varietäten und Sprachen sowie ihre Sprachgemeinschaften in Relation und diskutieren in diesem Zusammenhang ihre Dialekt-, Areal- und Sprachgrenzen. Dabei geht es nicht nur um die horizontalen Dialekt- und/ oder Sprachgrenzen, die zwischen zwei Dialekt- und/oder Sprachgebieten aufzufinden sind, sondern auch um die funktionalen Grenzen zwischen Funktionsbereichen (Domänen) von Varietäten und Sprachen in der gesellschaftlichen Interaktion, die in dem sozial strukturierten Raum vertikal (zwischen ,oben' und ,unten') verortet sind. Die intralingualen Sprachgrenzen zwischen Varietäten werden in der Variationslinguistik, die interlingualen Sprachgrenzen zwischen Sprachen und die Strategien ihrer Überschreitung in der Interlinguistik (vgl. Kimura 2018, S. 73f.) untersucht. Den interlingualen Sprachgrenzen ist in urbanen Zentren (contact zones) mehrsprachiger Regionen, Länder oder Nationalstaaten sowie in ihren Grenzregionen (borderlands; siehe hierzu Klatt in diesem Band) zu begegnen. Die Frage nach den Strategien der Überschreitung solcher Grenzen stellt sich dabei auch im Falle der „Mehrsprachigkeit an der Grenze“ (Matras 2009, S. 47), womit die Favorisierung der Sprache der ökonomischen Macht gemeint ist, die sich in der Wirtschaftsdomäne ihrer Nachbarstaaten bemerkbar macht. 
Abschließend befasst sich der Beitrag mit dem Übergang von einer Sprache zur anderen, der in der Interaktion nicht statisch ist, sondern in der Zeit geschieht. Dabei kann man beim Codeswitching innerhalb eines Textes, Textfragments, Satzes oder (nach manchen) auch Syntagmas die Sprachen in der Regel klar separieren, während sich die ,trennscharfe“ Sprachgrenze beim Codemixing nicht nur in Hybridwörtern, sondern auch im Text verliert. Dem stellt der Beitrag die Kopräsenz von Sprachen und/oder ihren symbolischen Visualisierungen (Schrift, Flaggen etc.) im öffentlichen Raum oder auf Artefakten gegenüber. In der Sprachlandschaft entstehen im Leerraum zwischen den Repräsentationen von Sprachen interlinguale Sprachgrenzen bzw. werden sie auf den Artefakten durch die Verflechtung dieser Repräsentationen in einem Zwischenraum aufgelöst (vgl. Marx/Nekula 2015). Durch Verortung (oben vs. unten, Zentrum vs. Peripherie) und Größe dieser Repräsentationen von Sprachen bilden sich neben den Sprachgrenzen auch Hierarchien diesseits und jenseits der Grenze heraus bzw. werden sie durch die Ausgestaltung dieser Repräsentationen auf den Artefakten aufgehoben. So lässt die Sprachlandschaft in der Repräsentation der Sprachen die horizontalen und vertikalen Sprachgrenzen und die horizontale und vertikale Strukturierung mehrsprachiger Gesellschaften erkennen.

\section{Intralinguale Grenzen}

Die Sprachgrenzen zwischen Varietäten einer Sprache wie Dialekte oder Slangs werden im Rahmen der Variationslinguistik behandelt. Die Dialektologie fokussiert primär auf die Sprachgrenzen bzw. Dialektgrenzen zwischen territorialen Dialekten auf der horizontalen Achse, d. h. nicht in Relation zur Standardvarietät, die auf der vertikalen Achse als ,höher ' gelten kann. Die Dialektologie, die die „,areale [...] Distribution sprachlicher Merkmale“ (Auer 2005, S. 149) untersucht, geht dabei den Fragen nach, wie sich die Gestalt einer Sprache in einem Territorium ausformt, ob darin von den Sprecher*innen einer Sprache territoriale Varietäten (Dialekte, Regiolekte) herausgebildet werden und wie sich die Dialekte einer Sprache anhand von linguistischen Merkmalen systematisch beschreiben, von anderen Dialekten abgrenzen und u.a. auch vermittels Repräsentation ihrer Grenzen in Sprachkarten und Sprachatlanten darstellen lassen.

Die klassische Erklärung der Vielfalt der sprachlichen Phänomene im geografischen Raum geht auf den Indogermanisten Johannes Schmidt (1872) zurück. Sie setzt in Anlehnung an die Physik davon voraus, dass sich sprachliche Innovationen im Raum wellenartig verbreiten, weshalb sie Wellentheorie genannt wird. In der Wellentheorie geht man dabei von den Annahmen eines Zentrums sprachlicher Innovation und der Peripherie ihrer allmählichen Ausbreitung aus, die in Bezug auf den Raum konsekutiv geschieht, in Kombination mit anderen Innovationen simultan erfolgen kann. Bei der allmählichen Ausbreitung verliert dabei die Innovation an Intensität und Durchschlagskraft bzw. wird sie zusammen mit der menschlichen Bewegungsfreiheit an natürlichen Barrieren und wechselnden politischen Grenzen angehalten oder dadurch verlangsamt. Territorien, die von solchen Innovationen und dem damit verbundenen Sprachwandel nicht erfasst sind und in denen es sozusagen beim Alten bleibt, gelten als Reliktgebiete. In diesem Sinne gelten etwa die ostmährischen Dialekte des Tschechischen als archaisch, weil darin die Umlautveränderungen ' $a>\check{e}$ (12.-13. Jh.) und ' $u>i$ (14. Jh.) sowie die Diphthongierungen $\dot{u}>$ ou (14. -15. Jh.) und $y$ > ej (15. -16. Jh.) nicht durchgeführt wurden, die sich in den böhmischen Dialekten des Tschechischen von Prag aus ausbreiteten und für diese charakteristisch geworden sind (vgl. Lamprecht et al. 1986). 
Die Grenzen der Ausbreitung solcher Innovationen bzw. Grenzen zwischen linguistischen Variablen, wie $i k \times i c h$ auf der (Uerdinger) ik-ich-Linie oder dat $\times$ das auf der (Sankt Goarer) datdas-Linie, die man in der Fläche durch Erhebung mittels Fragebögen feststellen, in eine Karte projizieren und darin abbilden kann, werden als Sprachgrenzen verstanden und können als „Isoglosse“ bezeichnet werden (vgl. Händler/Wiegand 1989). Diese Linien bzw. Isoglossen, die dialektale Kontinua aufteilen, sind also als sprachwissenschaftliche Konstrukte zu verstehen, die die Grenzen zwischen dem Ausbreitungsgebiet der einen und dem Reliktgebiet der anderen Variante einer Variablen repräsentieren und diese Gebiete auf der Basis der gesammelten Daten in der kartografischen Darstellung statisch trennen. Über Isoglossenbündel, so die Erwartung der Dialektologie, lassen sich dann Dialektgrenzen territorialer Dialekte bestimmen, die sich jeweils durch ein zusammenhängendes System von phonologischen und grammatischen Merkmalen auszeichnen. So stimmt die (Benrather) maken-machen-Linie weitgehend mit der ik-ichLinie, der dat-das-Linie, der dorp-dorf-Linie und der hebben-haben-Linie überein, wodurch dieses „Linienbündel“ (Bach 1969, S. 80) bei der Bestimmung der Grenze der niederdeutschen Dialekte eine wichtige Rolle spielt.

Die Entstehung solcher Linien und Linienbündel, die sich in den Repräsentationen in Sprachkarten und -atlanten zeigen, erklärt die klassische Dialektologie aus der Begrenzung der „Freizügigkeit“ (ebd., S. 81) der Menschen, die sich aus den physischen Begrenzungen im geografischen Raum, wie Flüsse, Bergzüge oder Sumpfgebiete, ergibt und durch staatliche Organisationsräume, wie Landes-, Bistums- oder Staatsgrenzen, gegeben wird. Eine solche Begrenzung verhindert den Verkehr, den Austausch und die gegenseitige sprachliche Akkommodation zwischen den Sprecher*innen derselben Sprache und verursacht Divergenz, d.h. die unterschiedliche Entwicklung und Ausdifferenzierung der Sprache diesseits und jenseits der ,natürlichen‘ oder politischen Grenze. Durch den Hinweis auf die Kongruenz zwischen den Sprachgrenzen und den politischen Staats- und Landesgrenzen sowie der „naturräumlichen Gliederung“ (König 1978, S. 143) werden der Raum und seine Grenzen, die Sprachgrenzen schaffen, als physisch gegeben gesetzt, während die Sprachgrenzen dadurch naturalisiert werden. Wenn sich also etwa Werner König (ebd., S. 141) auf „relativ starke Isoglossenbündelungen $[. .$.$] am Lech“ bezieht, führt er die Dialektgrenzen auf die scheinbar objektiv gegebenen$ Grenzen zurück, die für die Entstehung der Sprachgrenzen ursächlich verantwortlich seien. Dies gelte auch für politische Grenzen, die Sprachgrenzen schaffen (vgl. Schnabel 2006). In Anlehnung an den Ausdruck „Wasserscheide“ wird in ähnlichem Sinne von der ältesten „Sprachscheide“ auf der Linie Humber-Lune-Ribble gesprochen, die weitgehend mit der „Grenze zwischen den angelsäch[sischen] Königreichen Nordhumbriens und Merciens“ (Viereck/Viereck/Ramisch 2002, S. 91) zusammenfällt.

Die Erwartung, dass man so „klare Dialektgrenzen“ finden könnte, erwies sich aber als „irrig“ (Wenker 1888, S. 190; zit. nach Auer 2005, S. 152), denn selbst am Lech oder auf der Benrather Linie weichen die Isoglossen voneinander $a b$, des Öfteren sind sie weit gefächert oder anders ausgeformt, was man dadurch zu erklären versucht, dass die „Übergangszonen zwischen zwei Großdialekten [...] sehr breit sein“ können (König 1978, S. 141). Hinzu kommt, dass es auch unterschiedliche Ausbreitungszentren und -richtungen von Innovationen gab. Phonologische Innovationen erfassen außerdem keineswegs zwangsläufig den gesamten Wortschatz einer Sprache, sondern setzen sich über wortweise Umphonologisierungen durch, die sich über längere Zeitspannen und unter Umständen nie vollständig vollziehen. Am Ende vermag aber „niemand genau anzugeben [...], in wieviel Eigenheiten sich zwei Sprachgebiete unterscheiden 
müssen, damit man von zwei Dialekten sprechen kann“ (ebd., S. 141). So kommt es doch auf die bewusst reflektierten Merkmale an, die die Sprachgemeinschaft als ,Schibboleths“ (markante sprachliche Trennmerkmale) reflektiert, ohne dass sie trennscharf auf die geografische Grenze im physischen Sinne bezogen werden müssten. So orientieren sich linguistische Laien (Sprachbetrachter*innen), die gebeten werden, Dialekte auf Karten einzutragen, an geglaubten urbanen Zentren dieser Dialekte, anstatt die Nachbardialekte durch Sprachgrenzen voneinander abzugrenzen (vgl. Auer 2005, S. 152f.).

Hier setzt die Kritik an der klassischen Dialektologie an, welche den Raum und seine Grenzen nicht als geografisch bzw. physisch gegeben sehen will, sondern als Produkt von sozialen und diskursiven Praxen versteht. ${ }^{1}$ In kritischer Absetzung von der klassischen Dialektologie und ihrer Auffassung des Raums und dessen Grenzen sieht Auer (2005) in Anlehnung an Georg Simmel den Raum und seine Grenzen als Ergebnis mentaler Prozesse an, wobei die Sprache durch ihre Sprachbetrachter*innen und -nutzer*innen erst und eben in Bezug auf solche mentalen Grenzen überformt wird.

Auer (2005) konkretisiert dies anhand der Entwicklung von Sprachphänomenen in den Übergangsdialekten an der deutsch-niederländischen, deutsch-luxemburgischen, deutsch-französischen, deutsch-schweizerischen und deutsch-österreichischen Grenze. Dabei geht er von zwei Annahmen aus: Erstens, die politischen Grenzen sind nach dem Zweiten Weltkrieg im westeuropäischen Kontext physisch sehr wohl durchlässig, sodass eine gegenseitige sprachliche Akkommodation und damit eine parallele Entwicklung an beiden Seiten der politischen Grenze - anders als zwischen dem Ost- und Westblock während des Kalten Kriegs - durch den kleinen Grenzverkehr möglich sein müsste. Zweitens, „die alten Grenzen [des Nationalstaates] hinterließen ihre mentalen Spuren im kulturellen Gedächtnis der Bevölkerung und in ihren ethno-dialektologischen Landkarten“ (ebd., S. 162). Die Folge der Selbstverortung der Dialektnutzer*innen innerhalb des jeweiligen Nationalstaates führt an der niederländisch-deutschen Grenze zur Annäherung an die jeweilige Standardsprache und damit auf der Ebene der lokalen Dialekte zur Divergenz diesseits und jenseits der deutsch-niederländischen Staatsgrenze (vgl. Smits 2011), wodurch sich das niederfränkische Dialektkontinuum auflöst. Die Divergenzen im ehemaligen Dialektkontinuum sind also nicht durch physische Begrenzungen der Kommunikation, sondern durch die mentale Orientierung der Sprecher*innen an nationalen Raumkonzepten zu erklären.

Ähnlich verläuft die Entwicklung auch bei den Übergangsdialekten entlang der deutsch-luxemburgischen Grenze. Die Divergenz bei den Übergangsdialekten an der deutsch-französischen Grenze ist dagegen das Ergebnis einerseits der Annäherung der deutschen Dialekte an den deutschen Standard und andererseits auf französischer Seite das Ergebnis der Wahrnehmung der Übergangsdialekte als nichtdeutsche Regionalsprache, die sich in diesem Sinne ohne Ausgleich mit den Nachbardialekten weiterentwickelt (vgl. auch Auer 2015). An der deutsch-schweizerischen Grenze geht die Divergenz auf die unterschiedliche Repertoirestruktur deutscher Varietäten zurück: Das Prestige des Schweizerdeutschen gegenüber der negativen Bewertung des Standarddeutschen in der Schweiz führt dazu, dass die Übergangsdialekte auf der schweizerischen Seite als Dialekte stabil bleiben, während sie sich auf der deutschen Seite in Richtung des südwestdeutschen Regiolekts entwickeln. Bei den deutschen Dialekten entlang der deutsch-österreichischen Staatsgrenze ist schließlich durch die Nutzung derselben

1 Zur Raumpraxis, Raumrepräsentation und Repräsentationsräumen nach Henri Lefebvre (2006) und Verbindung des Ansatzes mit der Sprache vgl. Busch (2017) oder Marx/Nekula (2015). 
Standardsprache keine Divergenz, sondern eine Parallelentwicklung zugunsten regiolektaler Formen festzustellen, die allerdings entsprechend von München oder Wien aus geprägt sind. Diese Entwicklungen haben also, um dies noch einmal deutlich zu machen, mit der vor allem mentalen und nicht mit der geografischen Grenze oder der physischen Begrenzung der Sprachnutzer*innen zu tun, wobei man solche Vorstellungen über die Varietäten und Sprachen als Sprachideologie(n) fassen kann (vgl. etwa Woolard 1998), die die sozialen Normen in die sprachlichen übersetzen.

Noch eindringlicher zeigt sich die Bedeutung der Vorstellungen über den sozialen Raum und seine Strukturierung und Begrenzung für die Ausprägung bzw. Auflösung funktionaler Sprachgrenzen auf der vertikalen Achse, was man anhand der Etablierung, Wahrung und schrittweisen Auflösung der sogenannten Diglossie in der Schweiz und in Tschechien konkretisieren kann. Nach der klassischen Definition von Charles A. Ferguson (1959, S. 336) ist Diglossie

"a relatively stable language situation in which, in addition to the primary dialects of the language (which may include a standard or regional standards), there is a very divergent, highly codified (often grammatically more complex) superposed variety, the vehicle of a large and respected body of literature, either of an earlier period or in another speech community, which is learned largely by formal education and is used for most written and formal spoken purposes but is not used by any sector of the community for ordinary conversation“.

Die streng kodifizierte Varietät, die in der Schule erworben wird, kann man in Bezug auf die Schweiz mit hochdeutscher Standardsprache verbinden, der Schwyzerdütsch als (informell) gesprochene Varietät funktional gegenübersteht. Diese Diglossie zwischen dem Standard und dem Dialekt hat sich in der Schweiz aus dem Bedürfnis der ,Abgrenzung' gegenüber dem Deutschen und Deutschland im Zuge der Nationsbildung entwickelt (Giger 2003, S. 90, 94). Die Sprachsituation in der Schweiz unterscheidet sich dadurch von der Sprachsituation in Deutschland und Österreich, für die - jedenfalls im ober- und mitteldeutschen Raum - vertikal ein Kontinuum von Varietäten zwischen Nonstandard und Standard charakteristisch ist. In diesem Sinne hebt Georg Kremnitz (2005) hervor, dass die diglossische Sprachsituation „zu einer Funktion der sozialen“ Situation wird, „wobei eben sprachliche und soziale Trennlinien zusammenfallen“ (ebd., S. 160), während Markus Giger (2003, S. 83) diese „Trennlinien“ durch das Konzept der „komplementären“ sozialen Räume auf vertikaler Achse fasst, für die unterschiedliche Varietäten charakteristisch sind.

Mit der funktionalen Ausdifferenzierung und Komplementarität der Varietäten in Bezug auf Schriftlichkeit und Mündlichkeit bzw. formale Kommunikation in öffentlichen Domänen und informelle Kommunikation in der Privatsphäre und im Alltag entsteht also eine vertikal gedachte funktionale Sprachgrenze. Die Wahl von Schwyzerdütsch oder hochdeutscher Standardsprache gilt damit im Sinne von John J. Gumperz als Kontextualisierungshinweis (mehr dazu Auer 2013, S. 164-174), der die informelle oder formelle Kommunikation markiert.

Allerdings lässt sich nach Iwar Werlen (2004) in der Schweiz eine schrittweise Schwächung der Sprachgrenze bzw. „Demarkation“ (Kremnitz 2005, S. 163) zwischen Hochdeutsch und Schwyzerdütsch feststellen, die Werlen u.a. an der steigenden Zahl der Schulen mit Schwyzerdütsch als Unterrichts-, wenn auch nicht Schriftsprache festmacht und die auch Parallelen in der Kommunikation in Parlament, Bundesverwaltung, Kirche, Gericht, Armee, offiziellen Ansprachen oder elektronischen Medien hat (vgl. Giger 2003, S. 88). Diese ließe sich mit 
dem Wandel von Vorstellungen korrelieren, die sich auf den sozialen Raum, seine vertikale Organisation und die Komplementarität und Trennung von ,oben' und ,unten' beziehen. Die Schwächung der Diglossie in der Schweiz kann also als Ergebnis der „Tendenz zum Abbau gesellschaftlicher Normen, zur ,Informalisierung“ der Gesellschaft“ (ebd., S. 94) verstanden werden, die durch das niedrige Prestige des gesprochenen Hochdeutsch als Ergebnis des Abgrenzungsbedürfnisses dem ,Binnendeutschen“ gegenüber begünstigt wird. Die Lockerung und Verschiebung der imaginierten sozialen Grenzen lockert und verschiebt also auch die funktionale Sprachgrenze von zuvor komplementären Varietäten.

Einem ähnlichen, wenn auch anders ausgeprägten Fall kann man in Tschechien begegnen. Hier geht Neil Bermel (2010) von einer postdiglossischen Situation aus und ruft eine vorangegangene diglossische Situation ab, in der dem Gemeinböhmischen (obecná čeština, im böhmischen Teil Tschechiens), das von den Angehörigen der Sprachgemeinschaft im Alltag neutral genutzt wurde, das Standardtschechische als eine kodifizierte Varietät gegenübersteht, die in der Schule vermittelt und im geschriebenen und formalen mündlichen Ausdruck genutzt wurde. ${ }^{2}$ Da sich Diglossie per definitionem durch Komplementarität und eine funktionale Sprachgrenze auf der vertikalen Achse auszeichnet, markierte darin das Codeswitching von Standardtschechisch zu Gemeinböhmisch den (situativen oder metaphorischen) Übergang von der formellen zur informellen Kommunikation und das Codeswitching von Gemeinböhmisch zu Standardtschechisch den Übergang von der informellen zur formellen Kommunikation (am Beispiel aus den 1920er-Jahren ausgeführt in Nekula 2000).

Die Auflösung der Akzeptanz für die Organisation und Regulierung des sozialen Raums in der Richtung von oben nach unten, die sich in Tschechien im Jahre 1993 in der breiten gesellschaftlichen Auflehnung gegen die Disziplinierung der Sprachgemeinschaft durch eine noch vor der Wende vorbereitete Orthografiereform manifestierte (im Detail vgl. etwa Bermel 2007), dürfte durch das Ende der kommunistischen Diktatur lediglich verstärkt worden sein. Die Auflösung der Trennung von oben und unten sowie von Formalität und Informalität dürfte aber bereits früher eingesetzt haben und durch den Regimewechsel und die neuen elektronischen Medien nur verstärkt worden sein. Der Wandel der sozialen Normen geht dabei - vermittelt über entsprechende Sprachideologien - mit dem Wandel von sprachlichen Normen einher - in diesem Falle mit der schrittweisen Auflösung bzw. Normalisierung der Diglossie. So drängt das Gemeinböhmische immer mehr in die Domänen des Standardtschechischen vor, in den elektronischen Nachrichten und selbst in der Belletristik wird das Gemeinböhmische teilweise auch jenseits der direkten Rede genutzt. In der Interaktion gibt es dann kein motiviertes Codeswitching, das situativ oder metaphorisch unterschiedliche Kontexte bzw. damit verbundene Themen (formell vs. informell) markieren würde, sondern vielmehr ein Codemixing zwischen Standardtschechisch und Gemeinböhmisch innerhalb eines „minimalen Sprechakts“ (Giger 2003, S. 92), in dem das Fehlen der klaren Sprachgrenze im Dialog auch die Auflösung

2 Dabei lasse ich die Diskussion beiseite, ob man es im tschechischen Fall überhaupt mit Diglossie zu tun hat (vgl. Hronek/Sgall 1992, S. 215f.; Giger 2003, S. 92; Bermel 2014). Diese Zweifel werden durch gute Argumente genährt. So wird zwar auch Standardtschechisch erst in der Schule erworben, durch die Nähe der betreffenden Varietäten ist aber Standardtschechisch Kindern bereits im Vorschulalter gut verständlich. Auch beschränkt sich die Verwendung des Gemeinböhmischen auf Böhmen und damit auf zwei Drittel der Tschechischsprecher*innen, während ein Drittel der Tschechischsprecher*innen, nämlich diejenigen, die in Mähren aufgewachsen sind und leben, anstelle des Gemeinböhmischen andere Varietäten in einem Kontinuum verwenden. Die Rolle des Gemeinböhmischen in der Belletristik, die die Diglossie ebenfalls in Frage stellt, wird im Haupttext angesprochen. 
der klaren funktionalen Sprachgrenze in der Sprachsituation offenbart (zum interlingualen Codeswitching s. Abschnitt 5). ${ }^{3}$

Angesichts der in diesem Beitrag eingeführten Unterscheidung zwischen intralingualen und interlingualen Sprachgrenzen muss nun festgehalten werden, dass das Verständnis einer Sprachgrenze als intralingual oder als interlingual davon abhängt, ob das betreffende Sprachsystem als eigenständige Sprache oder als eine Variante oder Varietät einer Sprache angesehen wird. Wenn also ein deutschsprachiger Schweizer feststellt, dass Deutsch nicht seine Muttersprache sei (vgl. Giger 2003, S. 93), hebt er damit seinen Dialekt auf die Ebene der Sprache. Und wenn Franz Kafka im Februar 1912 den „Einleitungsvortrag über Jargon“ hält, stuft er Jiddisch auf die Ebene einer Varietät des Deutschen herab. Von solchen Beispielen gibt es eine Reihe. So auch bei der Vereinigung oder Spaltung von Sprachen. Während die Ideologie des Tschechoslowakismus in den 1920er-Jahren versucht hat, Tschechisch und Slowakisch als zwei Varianten einer tschechoslowakischen Sprache miteinander zu verknüpfen und ihren Abstand in der Orthografie zu verringern (vgl. Marti 1993), wurden die dialektal basierten Varianten des Serbokroatischen in den 1990er-Jahren von Sprachplanern in die Sprachen Bosnisch, Kroatisch und Serbisch aufgespalten und ihre Distanz systematisch ausgebaut (vgl. Raecke 2003; Vuković i.E.). Roland Marti (i.E.) zeigt wiederum, wie der Versuch, das durch Verbote im Dritten Reich angeschlagene Niedersorbisch neben Obersorbisch als Varietät des Sorbischen zu verstehen und die interlingualen nieder- und obersorbischen Grenzen - durch gemeinsame Kodifizierung - als intralinguale Grenzen umzudeuten, der kleinen slawischen Sprache in den 1950er-Jahren einen Todesstoß verpasst hat, der durch spätere Rücknahme der Orthografiereform, Wiederherstellung der interlingualen Grenze und Revitalisierungsmaßnahmen kaum zu stoppen ist. Wenn also im folgenden Abschnitt von interlingualen Grenzen die Rede ist, ist also stets zu berücksichtigen, dass die Annahme gegeneinander abgrenzbarer ,Sprachen“ Ergebnis sozialer Auseinandersetzungen und mentaler Zuschreibungen ist.

\section{Interlinguale Grenzen}

Das Fehlen der theoretischen Auseinandersetzung mit dem Raum in der Sprachwissenschaft führt Auer (2005, S. 150) auf die naive Vorstellung des geografischen Raums und seiner als ,natürlich“ verstandenen Grenzen (s. oben) sowie auf die Nachwirkung der „nationalstaatliche[n] Ideologeme [...], die [...] eine ,natürliche` Bindung (und notwendige Korrelation) zwischen Nation, Territorium und Sprache unterstellen “, zurück.

So stellt etwa der tschechische Sprachwissenschaftler Bohuslav Havránek (1934, S. 211f.) bei der Abgrenzung des Tschechischen und Polnischen in einer enzyklopädischen Darstellung der tschechisch-polnischen Übergangsdialekte zwar zunächst die mannigfaltigen Grenzziehungen anderer Sprachwissenschaftler*innen dar und zeichnet die südliche und nördliche Grenze des Übergangsgürtels ein, am Ende richtet er aber die tschechisch-polnische Sprachgrenze trennscharf nach der tschechisch-polnischen nationalen Grenze aus, die in der kartografischen Darstellung die Ergebnisse der Volkszählung im Jahre 1921 abbildet. Im Rahmen des staatlich

3 Die beiden Begriffe werden sehr unterschiedlich aufgefasst. Beispielsweise spricht Shana Poplack (2004) vom Codeswitching, d.h. dem Wechsel zwischen zwei Kodes bzw. dem Übergang von einer Sprache zur anderen, und zwar sowohl innerhalb eines Satzes oder Redebeitrags bzw. einer Äußerung als auch an der Grenze zwischen zwei satzartigen Segmenten. Statt des Codeswitching diskutiert Pieter Muysken (2000) das Codemixing, d.h. die Mischung von Kodes (Sprachen) und seine drei Formen (Insertion, Alternation, kongruente Lexikalisierung), wobei - abgesehen von den Übergängen zwischen den angesprochenen Gruppen - eigentlich nur die Alternation in der Sprachkontaktforschung als Codeswitching gilt (mehr dazu Riehl 2014, S. 21-35). 
finanzierten Projektes Sprachatlas des Deutschen Reiches erhärtet die deutsche Dialektologie durch ihre Methoden und wissenschaftliche Autorität die Vorstellung der Einheit der deutschen Nation, die „auf der Verschiedenheit ihrer Stämme“ basiere (Auer 2005, S. 151), und bindet die exterritorialen Enklaven an das Heimat- oder Kernland. Im Dritten Reich werden in den Darstellungen des Sprachgebiets des deutschen Volkes auch unter Rückgriff auf den Standard, der dieses Gebiet überwölbt, die mehrsprachigen deutsch-slawischen Mischgebiete in West- und Ostpreußen, in der Lausitz oder in Böhmen und Mähren entsprechend verdrängt (ebd., S. 153). Die rhetorische Unterwerfung der Sprachwissenschaft unter die herrschenden Ideologien bis 1945 spricht Klaas-Hinrich Ehlers (2010, S. 75-150) an.

Diese Ideologeme, die nicht nur in der sprachwissenschaftlichen (diskursiven), sondern auch in der politischen (sozialen) Praxis zu Grenzverschiebungen und Bereinigungen der Sprachgebiete und deren Sprachgrenzen führten, offenbaren sich u.a. auch im Zusammenhang mit den Vorstellungen über die ,innere deutsch-tschechische Sprachgrenze innerhalb der böhmischen Länder. Dabei geht es weniger darum, dass sich ihr Verlauf seit der Etablierung während der großen deutschen Kolonisierung im 12./13. Jahrhundert bis zu ihrer Auflösung durch die Zwangsaussiedlung in den Jahren 1945-1947 veränderte. Vielmehr geht es darum, dass das Attribut in der Wortgruppe ,innere Sprachgrenze deutlich macht, dass für die Zeitgenossen die Übereinstimmung der sprachlichen und der politischen Grenze den Normal- bzw. den Idealzustand darstellte. Eine Abweichung davon war daher durch das Attribut wertend markiert. So dachten die Protagonist*innen der tschechischen ethnonationalen Agenda an eine Ausdehnung der Sprachgrenze und damit auch des autonomen und homogen verstandenen ethnonationalen Raums bis zur Landesgrenze Böhmens und Mährens, die durch Grenzgebirge und -flüsse naturalisiert zu sein schien (mehr dazu vgl. Haslinger 2010). Auf der anderen Seite dachte man an die Schaffung einer Verwaltungsgrenze entlang der bestehenden inneren Sprachgrenze, mit der die politische Autonomie der dominant deutschsprachigen Gebiete bzw. die Föderalisierung des böhmisch-mährischen Territoriums geschaffen werden sollte. Die politische Grenze entlang der von deutscher Seite imaginierten deutsch-tschechischen Sprachgrenze wurde später mit der Abtretung der Sudetengebiete geschaffen, was eine Vertreibung und Verdrängung der tschechischsprachigen Bevölkerung hinter diese Grenze zur Folge hatte.

Diese Vorstellung der territorialen, horizontalen Sprachgrenze geht Hand in Hand mit der Vorstellung der sozialen, vertikalen Sprachgrenze. Die Ausweitung der Landessprachen von nur Tschechisch auf Deutsch und Tschechisch in Böhmen (1627) und in Mähren (1628), die anschließende Zentralisierung des Habsburgerreiches und schließlich die theresianischen und josephinischen Reformen, die die dominante Stellung von Deutsch im Schulwesen und in der Verwaltung ausbauten und absicherten, schufen in den böhmischen Ländern eine Außendiglossie und eine funktionale Sprachgrenze mit der Dominanz des Deutschen im öffentlichen Raum und in der formalen Interaktion. ${ }^{4}$ Das "Gelenk“ (Kremnitz 2005, S. 160) zwischen den vertikal gedachten und funktional komplementären Sprachräumen waren im langen 19. Jahrhundert die Bilingualen, die in Mischehen und/oder durch Bildung die andere Sprache erworben haben. Da Deutsch sozialen Aufstieg oder soziale Stabilisierung versprach, geschah dies weitgehend unidirektional. Im Laufe der zweiten Hälfte des 19. Jahrhunderts wurde im Zuge der tschechischen ethnonationalen Agenda an der funktionalen Grenze dieser Außendiglossie gezerrt und das autonome tschechische Schulwesen sowie andere kulturelle und wirtschaftliche

4 Nach Heinz Kloss (1966, S. 138) kann man zwischen Innendiglossie, die zwischen Varietäten einer Sprache entsteht, und Außendiglossie, die sich zwischen zwei Sprachen herausbildet, unterscheiden. 
Institutionen ausgebaut (vgl. Überblick in Stöhr 2010). Dadurch wurden die funktionalen Grenzen des Tschechischen verschoben. Schließlich wurde die Außendiglossie bereits vor der Entstehung der Tschechoslowakei im Jahre 1918 weitgehend abgebaut bzw. - mit Kremnitz (2005, S. 161) formuliert - „normalisiert“. Die Expansion des Tschechischen lässt sich an der Zulassung von Tschechisch als äußere Amtssprache (1880) und an der Teilung der Prager Karlsuniversität (1882) in eine deutsche und tschechische festmachen, wobei die Zulassung von Tschechisch als innere Amtssprache, die die Badeni-Reform von 1897 vorsah, zwar de jure zurückgenommen, nach Simona Švingrová (2010) aber in öffentlichen Institutionen um 1910 de facto praktiziert wurde. Auch die nicht zurückgenommenen Sprachverordnungen sowie die Sprachgesetze und -verordnungen der 1920er-Jahre wirkten freilich zeitversetzt, sodass die tatsächliche Sprachwirklichkeit der intendierten Sprachwirklichkeit hinterherhinkte und die vertikale Sprachgrenze keine scharfe Trennlinie darstellte, wie dies Stefan M. Newerkla (1999) oder Mirek Němec (2009) für das Schulwesen und Jaroslav Kučera (1999) für die Verwaltung ausführen. Die entscheidende Rolle der mentalen Raum- und Grenzvorstellungen für den Wandel - die Verschiebung, Einschätzung und Ausgestaltung - der interlingualen Grenzen dürfte hiermit dennoch deutlich geworden sein.

\section{4. Überschreitung von interlingualen Grenzen}

Die Möglichkeiten, interlinguale Grenzen kommunikativ zu überschreiten, reflektiert u.a. Sonja Vandermeeren (1998), die im Kontext der internationalen Unternehmenskommunikation drei idealtypische Strategien unterscheidet und deren jeweilige Vor- und Nachteile abwägt. Die Form der internationalen Kommunikation, bei der die Kommunikationsteilnehmer*innen jeweils ihre Sprache verwenden und die Sprachgrenze mit Hilfe von Sprachvermittler*innen überschritten wird, nennt sie „Nicht-Adaption“ (ebd., S. 36f.). Die Sprachvermittlung erfolgt dabei ohne, oder - bei (passiver) Kenntnis der anderen Sprache - mit ein- oder beidseitiger Kontrolle durch die Kommunikationsteilnehmer*innen, für die vermittelt, also übersetzt und/oder (konsekutiv oder simultan) gedolmetscht wird. Diese Form der Kommunikation werde bei der Anbahnung und beim Ausbau von internationalen Kooperationen genutzt. Verwendung findet sie auch bei internationalen Konferenzen, Jugendbegegnungen (vgl. Marx/ Nekula 2014) oder auf politischer Ebene, auf der es bei internationalen Kontakten - wie bei bilateralen Verhandlungen oder im Europäischen Parlament - neben der Verständigung auch um die symbolische Symmetrie zwischen den Kommunikationsteilnehmer*innen geht. Etwa bei Gerichtsverfahren soll auf diese Weise wiederum die Wahrung des Rechts auf ein faires Verfahren für Betroffene, die die Verfahrenssprache nicht oder nicht vollkommen beherrschen, sichergestellt werden. Dabei offenbart sich allerdings auch die Asymmetrie zwischen den Angehörigen der Sprachgemeinschaft, die dafür entsprechende personelle und finanzielle Ressourcen zur Verfügung stellt, und denen, die auf diese Weise in ihr Sprachregime eingeordnet werden. Bei Verkettung mehrerer Dolmetsch- oder Übersetzungsprozesse im Falle weniger frequentierter Quellen- oder Zielsprachen wird die dazwischengeschaltete Sprache als Relaissprache bezeichnet, wobei dadurch zwei (oder mehrere) Sprachgrenzen bzw. ein ausgedehnter Translationsraum entstehen, der Zeit und Mittel kostet (zu Kosten der Nicht-Adaption in deutsch-tschechischer Unternehmenskommunikation vgl. Nekula 2002 bzw. Nekula/Šichová 2004).

Wenn bei der kommunikativen Überschreitung der interlingualen Grenze die Kenntnis der Sprache des jeweils anderen Gesprächspartners vorausgesetzt und diese Sprache auch ver- 
wendet wird, spricht Vandermeeren (1998, S. 36f.) von „Adaption“. Bei der symmetrischen Adaption nutzt jede/r aktiv seine Sprache, während sie/er imstande ist, die Sprache der/des Anderen passiv zu verstehen, wie dies in der Korrespondenz zwischen Franz Kafka und Milena Jesenská der Fall war. Bei der asymmetrischen Adaption wird die Sprache nur einer/s der Kommunikationsteilnehmer*innen (Partner*innen) genutzt. Der Erfolg beider Varianten hängt von dem Niveau ab, welches beim Erwerb der Fremd- oder Zweitsprache erreicht wurde, bzw. auch davon, inwieweit man die kommunikative Asymmetrie (vgl. Nekula 2002) berücksichtigt und die Verständigung durch die Wahl des Standards und die Reduktion des Tempos, der Komplexität sowie der Modalität fördert.

Die erste Variante wird des Öfteren etwa in der Kommunikation zwischen Tschechen und Slowaken angewendet, bei denen zumindest für die ältere und mittlere Generation passiver Bilingualismus vorausgesetzt werden kann, der in der 1970er und 1980er Jahren durch die Medien und teilweise auch in der Schule eingeübt wurde. Bei der Verwendung von verwandten Sprachen wie Tschechisch und Polnisch oder Deutsch und Niederländisch, bei denen man sich lediglich auf die gegenseitige Verständlichkeit verlässt, sowie beim unzureichenden Fremdoder Zweitsprachenerwerb kommt es zur Semikommunikation, d.h. zur aktiven Verwendung der eigenen und zur passiven Rezeption der anderen Sprache, bei der das Verständnis ungesichert ist und die Sprachgrenze nur fragmentarisch überschritten wird.

Die asymmetrische Variante der Adaption war - in der Transformationszeit des ehemaligen Ostblocks - etwa für die multinationalen Unternehmen in der Tschechischen Republik charakteristisch (vgl. Nekula et al. 2005a; 2005b; Nekvapil/Nekula 2006), die in irgendeiner Form in die Struktur von Unternehmen aus deutschsprachigen Ländern eingebunden wurden. Vor allem in großen Unternehmen mit ausländischen Manager*innen und Projekt- und Abteilungsleiter*innen wurde auf der Managementebene Deutsch (später auch Englisch) präferiert, während die Produktion weitgehend tschechisch geprägt war und ist, was in diesen Unternehmen zur Herausbildung einer Außendiglossie führte, die u.a. auch durch Festlegung der Firmensprache zugunsten des Deutschen (oder Englischen) stabilisiert wurde. Weitaus wichtiger sind aber für die Stabilisierung der funktionalen Sprachgrenze die von beiden Seiten akzeptierten Sprachideologien, wonach Deutsch eine große und relevante Sprache und Tschechisch lediglich eine kleine, schwer zu erwerbende, für die ausländischen Manager*innen nur temporär relevante Sprache sei (vgl. Nekvapil/Sherman 2013). Dabei werden die Sprachideologien mit dem mental vorgestellten sozialen Raum und seinen vertikal verorteten Grenzen verknüpft. Das ,Gelenk' an dieser vertikalen Sprachgrenze waren und sind mehrsprachige Mitarbeiter*innen auf der mittleren Managementebene, wobei ihr deutsch-tschechischer Bilingualismus unidirektional ausgeprägt ist. Mehrsprachig sind also überwiegend Mitarbeiter*innen mit Tschechisch als Erstsprache. Die Favorisierung der Sprache der ökonomischen Macht und die Entstehung der „Mehrsprachigkeit an der Grenze“ (Matras 2009, S. 47) in der Wirtschaftsdomäne von Nachbarstaaten, wodurch auch die horizontale Dimension dieser vertikalen Sprachgrenze angesprochen ist, trifft dabei nicht nur für Mitteleuropa zu, sondern lässt sich mit anderen Sprachkombinationen auch in Einflusssphären anderer politischer und/oder ökonomischer Großmächte feststellen.

Die internationale Kommunikation, bei der die interlinguale Grenze mit Hilfe einer dritten Sprache überschritten wird, wird als „Standardisierung“ (Vereinheitlichung) bezeichnet (Vandermeeren 1998, S. 46). Eine solche Überschreitung der Sprachgrenze(n) wird im gegebenen Kontext mit der Lingua franca verbunden, die - wie Englisch - eine globale oder - wie Rus- 
sisch oder Deutsch - eine glokale bzw. areale Reichweite hat, wobei sich die Bedeutung sowie territoriale und funktionale Reichweite der Lingua franca (Latein vs. Englisch) in der Zeit verändert (zum Englischen vgl. Sherman und Nekvapil 2018 oder Publikationen im Journal of English as a Lingua Franca).

Das gerade skizzierte Modell wurde für die internationale Unternehmenskommunikation entwickelt und spricht in diesem Rahmen idealtypische Strategien der Überschreitung der interlingualen Grenze an. In der realen Kommunikation werden diese idealtypischen Strategien kombiniert bzw. vermischt. Christoph Marx und Marek Nekula (2014) dokumentieren, dass etwa bei deutsch-tschechischen Jugendbegegnungen bei der Überschreitung der Sprachgrenze alle drei idealtypischen Strategien genutzt werden: Während der von der organisierenden Institution strukturierte Teil solcher Begegnungen von der Nicht-Adaption dominiert ist, kommen in dem nichtstrukturierten Teil Englisch als Lingua franca sowie Schulkenntnisse des Deutschen seitens der tschechischen Schüler*innen zum Einsatz.

Wie bereits angedeutet, wurde dieses Modell auch für die Beschreibung der Überschreitung der interlingualen Sprachgrenze bei der Mehrsprachigkeit an der Grenze übernommen. Dabei haben Nekula et al. (2005a; 2005b) auch Strategien der symbolischen Neutralisierung der kommunikativen Asymmetrie, die durch die asymmetrische Adaption entsteht, festgestellt. Durch die symbolischen Neutralisierungsstrategien (Einführung von Führungstandems, Zweisprachigkeit der Räume, eingeübte tschechische Ansprache der Belegschaft durch die Firmenleitung, die sonst kein Tschechisch verwendet, usw.) wird die kommunikative Asymmetrie symmetrisiert und die vertikale Anordnung des Raums und die vertikale Verortung der Sprachgrenze, die durch die kommunikative Asymmetrie produziert wird, in eine horizontale übersetzt. Prinzipiell wäre dieses Modell auch auf die Überschreitung der Sprachgrenzen zwischen Minorität und Majorität anwendbar, welche für die Migrant*innen (allochthone Minorität) in der ,westlichen Welt‘ zunächst mittels des Dolmetschens (Nicht-Adaption) und/oder Englisch als Lingua franca (Standardisierung) überschreitbar gemacht wird, bevor mit der Zeit die Adaption an die Zweitsprache einsetzt. Diese setzt bei einer autochthonen Minorität oder im Rahmen einer Sprachautonomie in der Regel mit der sekundären Sozialisation ein (zu Typen der sprachlichen Statusplanung vgl. Janich 2007). Um die Überschreitung der interlingualen Grenze(n) zu sichern, setzt man beim sprachlichen Föderalismus - wie in der Schweiz - auf die Erziehung zum bidirektional erworbenen Bilingualismus und zu einer möglichst symmetrischen sozialen Mehrsprachigkeit sowie auf die institutionelle Mehrsprachigkeit einschließlich des (bioder multidirektionalen) Bilingualismus der darin tätigen Mitarbeiter*innen. Das Letztere trifft auch für Länder mit institutionalisierter Mehrsprachigkeit wie Irland oder Finnland zu (zu Übersetzungsprozessen im habsburgischen Vielvölkerstaat vgl. Wolf 2013).

Goro Christoph Kimura (2018) stellt die bereits angesprochene Frage der Überschreitung der Sprachgrenzen (crossing linguistic borders) in den Kontext der Interlinguistik und der Grenzstudien (Border Studies) und präzisiert - auch mit Blick auf die Mischformen - die oben angesprochenen idealtypischen ,Konstellationen“ der Überschreitung der Sprachgrenzen am Material des deutsch-polnischen Grenzgebiets (borderlands). Dabei arbeitet er mit der Unterscheidung von Sprachen in native language, partner language, additional language und den damit verbundenen Strategien: 


\begin{tabular}{|l|l|l|}
\hline & common strategy & alternative strategy \\
\hline $\begin{array}{l}\text { native-language stra- } \\
\text { tegies }\end{array}$ & $\begin{array}{l}\text { language mediation } \\
\text { [Nicht-Adaption] }\end{array}$ & $\begin{array}{l}\text { receptive multilingualism } \\
\text { [symmetrische Adaption] }\end{array}$ \\
\hline $\begin{array}{l}\text { partner-language } \\
\text { strategies }\end{array}$ & $\begin{array}{l}\text { internal-language asymmetry } \\
\text { [asymmetrische Adaption] }\end{array}$ & partner-language symmetry \\
\hline $\begin{array}{l}\text { additional-language } \\
\text { strategies }\end{array}$ & $\begin{array}{l}\text { lingua franca } \\
\text { [Standardisierung] }\end{array}$ & Esperanto \\
\hline
\end{tabular}

Abbildung 1: Modell nach Kimura (2018, S. 74f.) mit deutschen Kommentaren von mir, in denen dieses Modell auf das Vandermeerens bezogen wird

Die grundlegenden Strategien von Kimuras Modell wurden bereits oben angesprochen und sind in der Tabelle in Klammern vermerkt. Mit der „partner-language symmetry“ wird eine besondere Form der symmetrischen Adaption bezeichnet, bei der in einem „foreign polyglot dialogue“ jeder aktiv die Sprache des Anderen verwendet (Kimura 2018, S. 77). Durchlässige Grenzen dehnen nach Barbara Alicja Jańczak (2018b, S. 89f.) die Sprachgrenze auf die Grenzregionen (borderlands) als „spaces of transition“ aus, für die „language switching“ (ebd., S. 92) charakteristisch sei.

\section{Grenzen in und Grenzüberschreitung durch Codeswitching}

In den Ausführungen oben ist bereits deutlich geworden, dass die intralingualen und interlingualen Grenzen auch in der sprachlichen Interaktion beim Codeswitching und Codemixing bilingualer Sprecher*innen sichtbar oder hörbar und in der Interaktion überschritten werden (zu Codeswitching und Codemixing siehe auch Anm. 3). In diesem Sinne spricht Yaron Matras (2009, S. 101) über „crossing boundaries“ im bilingualen Modus der Kommunikation. Nach Auer (1983, S. 52) bedeutet Codeswitching

„eine Veränderung der bisherigen Sprachwahl, sei es durch alle Teilnehmer, sei es durch einen von ihnen. An einem bestimmten Punkt im Ablauf der Konversation wird die etablierte Sprachwahl aufgekündigt und durch eine neue ersetzt bzw. zu ersetzen versucht. Codeswitching ist also ein Übergang von einer Sprache in die andere“.

Dieser Übergang markiert also eine Sprachgrenze, deren Ausprägung unter anderem davon abhängt, ob es sich um ein funktionales (bewusstes) oder ein nichtfunktionales (nicht bewusstes) Codeswitching handelt (vgl. Riehl 2014, S. 25-31), wobei im Weiteren eine Beschränkung auf das interlinguale Codeswitching erfolgt.

Beim funktionalen Codeswitching scheint es, als ob die Segmente trennscharf separiert werden könnten. In seinem Brief an Milena vom 25. bis 29. Mai 1920 wechselt Franz Kafka in dem Satz „Vor einigen Jahren war ich viel im Seelentränker (man̆as) auf der Moldau [...]“ (Kafka 1998, S. 21; Herv. M. N.) von einer Sprache zur anderen und übersetzt dabei mit Rücksicht auf seine Adressatin den deutschen Ausdruck für ein kleines Ruderboot in ihre Muttersprache. Von den Klammern abgesehen, weist die Grenze - in diesem Fall die Wortgrenze - zwischen beiden Segmenten, die zugleich eine interlinguale Sprachgrenze ist, keine Materialität auf. Neben einem solchem smooth codeswitching, wo der Übergang derart glatt und zugleich trennscharf verläuft, gibt es aber in der mündlichen Interaktion auch flagged 
codeswitching (Poplack/Sankoff 1988, S. 1176), wie etwa im Beispiel „(.) ähm tam (.) se tam () [= unverständliche Stelle] bylo hodně [dort (.) dort () gab es viel] ähm (.) gewalt“ (Magdalena, Juli 2006) zeigt. ${ }^{5}$ Infolge eines Wortfindungsproblems, das durch Verlangsamung des Tempos und Häsitation erkennbar wird und - sprecher*innenbezogen - mögliches Codeswitching ankündigt, das sich in diesem Fall am Ende auch vollzieht, entsteht durch die Häsitation ein (teilweise) sprachlich unspezifischer ausgedehnter materieller Übergang von einer zur anderen Sprache. Dieser kann auch Form eines metasprachlichen Kommentars haben, wie „jak se řekne äh česky scheidung, [= wie sagt man auf Tschechisch scheidung,]“ (Magdalena, Juli 2006).

Auch beim nichtfunktionalen Codeswitching hat der Übergang von einer zur anderen Sprache eine Materialität, wenn diese auch anders ausgeprägt ist. Dieser Übergang geschieht nicht bewusst und ist durch die Verwendung von sogenannten Triggerwörtern zu erklären, die den Wechsel von einer Sprache zur anderen auslösen. Dies ist beim Übergang vom Niederländischen zum Englischen in "Weet je [= Do you know] what she is doing?" (Riehl 2014, S. 24; kursive Herv. M. N.) der Fall. Das Wort wat, das im Niederländischen und Englischen weitgehend identisch lautet (homophon ist) und auch semantisch kongruent lexikalisiert ist, bildet hier ein ,Gelenk' zwischen beiden Segmenten und gehört beiden Sprachen an bzw. kann beiden angehören, weswegen der Wechsel unbewusst eingeleitet (getriggert) wird. Neben solchen kongruenten Lexikalisierungen, die in beiden Sprachen identische lautliche Form und Semantik haben, gehören zu den Triggern etwa auch Namen oder Lehnwörter.

Auch wenn dadurch beide Segmente miteinander verflochten sind, sind sie sonst jenseits des Triggers materiell und strukturell so verschieden, dass der durch den Trigger erfolgte Übergang von der einen in die andere Sprache als sprachlich ambivalente Grenze zwischen diesen Segmenten gelten kann. Dagegen wird diese bei der Selbststilisierung wie in „Druhý [= das zweite] Glas mám [= habe ich] in meinem Zimmer" (Kateřina, Studierende der Deutsch-Tschechischen Studien, Juli 2018 - Beispiel Krempaská 2019, S. 17, entnommen; Herv. M. N.) durch das Codemixing (translinguistic wording) bewusst verwischt. Bei den Herkunftssprecher*innen, bei denen es zu keiner vollkommenen Ausdifferenzierung des integrierten Sprachsystems gekommen ist, werden wiederum etliche lexikalische Einheiten der primären Sprache auch in der sekundär gewordenen Erstsprache, und zwar ohne eine Alternative, genutzt, d.h., diese gehören der einen wie der anderen Sprache an, wodurch die Herkunftssprache mit der Umgebungssprache verflochten ist. Bei einem solchen Codemixing ist ein funktionales (bewusstes) Codeswitching gar nicht möglich.

\section{Sprachen und ihre Grenzen in der Sprachlandschaft}

Von beiden Varianten des Codeswitchings, die in der mündlichen Interaktion in der Zeit geschehen, unterscheidet sich die visuelle Kopräsenz von Sprachen und ihren symbolischen Darstellungen im öffentlichen Raum bzw. auf den öffentlich verfügbaren Artefakten. Die Verteilung der Sprachen über eine Stadt oder eine Grenzregion (borderland) ist allerdings lediglich durch die Bewegung im Raum und damit auch in der Zeit erfahrbar, während das Codeswitching im gedruckten Text - etwa in Comics - visuell auch auf einmal erfassbar ist.

Das wissenschaftliche Interesse an der visuellen Nichtpräsenz und/oder Kopräsenz von Sprachen im öffentlichen Raum beginnt in den 1990er-Jahren. Analysiert werden die Verschriftli-

5 Zitiert aus den noch nicht veröffentlichten Aufnahmen des Autors mit einer Herkunftssprecherin des Tschechischen, wobei der Name geändert wurde; so auch im Folgenden. 
chung oder symbolische Visualisierung von Sprachen auf Straßen- und Orientierungstafeln, Ladenschildern und Schaufenstern, Inschriften und Aushängen an und in Institutionsgebäuden, auf Werbeplakaten usw., die sich mit dem Raum in einer Grenzregion, einer Stadt, einem Unternehmen oder einer Institution wie einer Behörde oder Schule verbinden und so eine Sprachlandschaft (linguistic landscape) unterschiedlicher Reichweite und Komplexität produzieren. Ausgehend von der Annahme, dass die Repräsentation von Sprachen im öffentlichen Raum mehrsprachiger Gesellschaften mit der Rolle ihrer Sprachgemeinschaften in der Gesellschaft korreliert, erwartet man von der Analyse von Sprachlandschaften Erkenntnisse nicht nur über den Status und das Verhältnis von Sprachen in der Gesellschaft, sondern auch über die Stellung und Beziehungen von Sprachgemeinschaften. Diese werden an der (Nicht-)Präsenz und Verortung (Zentrum vs. Peripherie z.B. in einer Stadt, Verteilung auf Funktionsräume wie Behörden vs. Gastronomie, außen vs. innen) und bei Kopräsenz an der Anordnung (oben vs. unten, links vs. rechts) und Ausgestaltung (Größe der Schrift, Hand- vs. Druckschrift) von Repräsentationen der Sprachen im öffentlichen Raum sichtbar.

Diese kann man als Ergebnis der Sprachpolitik bzw. des organisierten Sprachmanagements von oben in Wechselwirkung oder Konkurrenz mit dem einfachen (oder organisierten) Sprachmanagement von unten (vgl. Nekvapil 2009) verstehen, wie dies das Bild des polnisch-deutschen Ortsschilds im polnischen Schlesien mit amtlich hinzugefügter und durch eine/n Aktivist*in beschmierter deutscher Inschrift illustriert (s. Abb. 2).

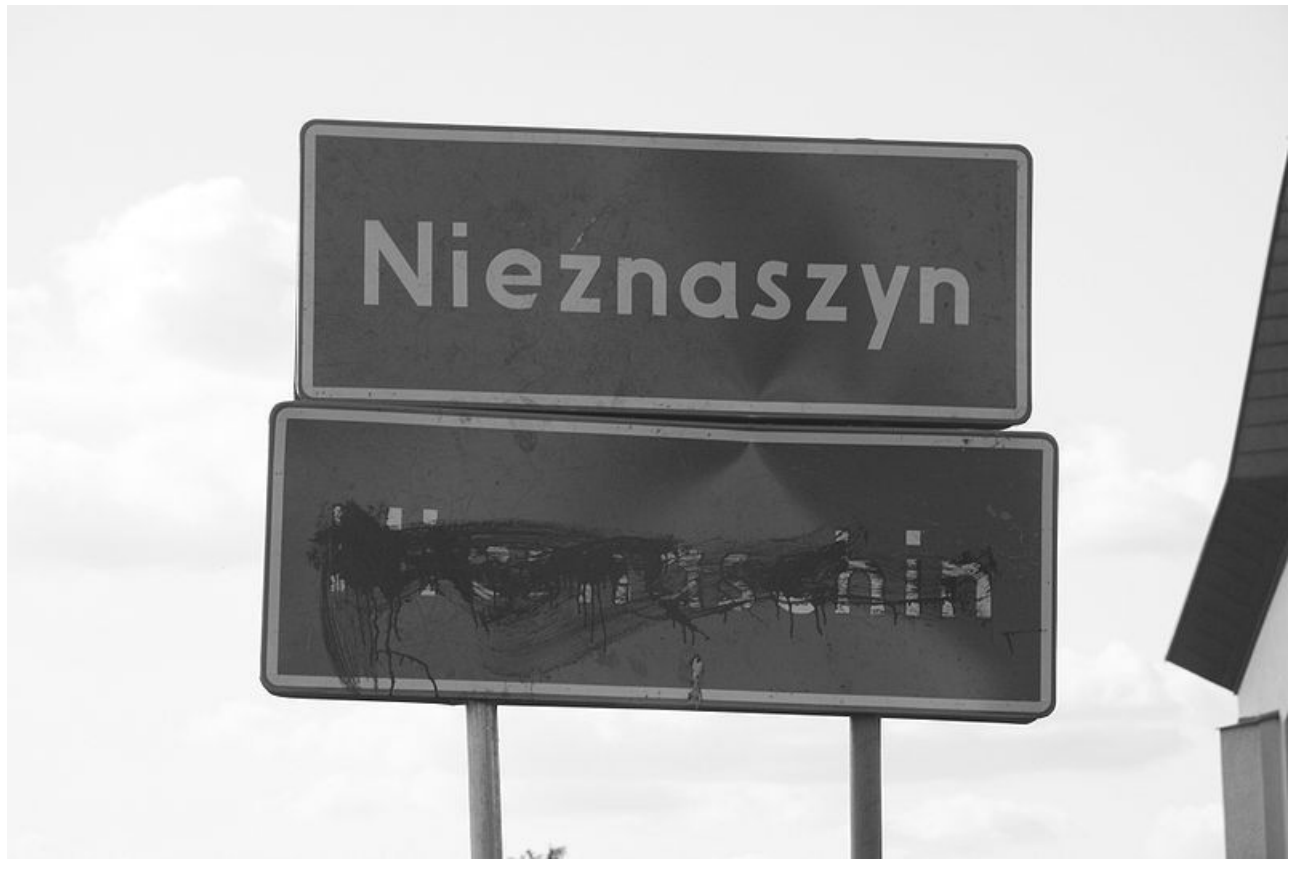

Abbildung 2: Nieznaszyn/Niesnaschin. Quelle: https://austria-forum.org/af/AustriaWiki/Deutsche_Minderheit_in_ Polen

In diesem Sinne beschreiben Bernard Spolsky und Robert Cooper (1991) die Sprachlandschaft und Sprachpolitik in Jerusalem, Sebastian Muth (2014) in Transnistrien, Jańczak (2018b) die linguistic borderscape in der deutsch-polnischen Grenzregion sowie Marx und Nekula (2015) 
in Organisationen an der deutsch-tschechischen Sprachgrenze, an der diese eine Übersetzungsarbeit leisten. Selbstverständlich geht es dabei nicht nur um die Mehrsprachigkeit einer Gesellschaft. Die japanisch-englische Zweisprachigkeit bzw. urbane Mehrsprachigkeit in Tokyo (vgl. Backhaus 2007) z.B. offenbart die Stadt als eine Metropole der globalen Wirtschaft und des globalen Handels. Neben der äußeren Mehrsprachigkeit kann auch die innere Mehrsprachigkeit (Standard vs. Dialekt) sichtbar werden.

Mit den mehrsprachigen Sprachlandschaften sind auch interlinguale Sprachgrenzen im Zwischenraum der kopräsenten visuellen Repräsentationen von Sprachen verbunden. Im Falle ihres Nebeneinanders werden diese Repräsentationen horizontal oder vertikal in Relation gesetzt und durch eine Sprachgrenze verbunden. Teilweise ist auch eine visuelle Verschmelzung der Sprachen bzw. Aufhebung der trennscharfen linearen Grenze, die sprachnationale Separation impliziert, festzustellen. Diese geschieht oft im Logo, der Bezeichnung oder den Artefakten mehrsprachiger Institutionen, Städte oder Regionen. So weichen Organisationen, die in der deutsch-tschechischen Kontaktzone aktiv sind und ihre Sprachgrenze überschreitbar machen, auf Latein aus, wie beispielsweise das Centrum Bavaria Bohemia, bzw. auf kongruente Lexikalisierungen wie Tandem, um die Grenzregion bzw. Translationsprozesse in der Kontaktzone jenseits der Abrufung der Sprachgrenze durch Zweisprachigkeit zu bezeichnen (zur deutschpolnischen Grenzregion vgl. Kimura 2018). Dabei setzen solche Grenzorganisationen bei der Überschreitung der interlingualen Grenze in der Regel auf die konsequente und nach außen getragene „tatsächliche“ Zweisprachigkeit von öffentlichen Texten und Auftritten (Marx/Nekula 2014, S. 64), die beide Zielgruppen ansprechen und zusammenführen sollen, wobei der Translationsraum dadurch im Sinne einer sprachlichen und nationalen Symmetrie symbolisch aufgeladen wird. Die dadurch praktizierte Überschreitung der interlingualen Sprachgrenze wird dabei symbolisch erst dadurch möglich, dass diese vermittels der Zweisprachigkeit symbolisch geschaffen wird. Dabei wird neben der Zweisprachigkeit von Geschäftsräumen, Homepages, Plakaten, Texten oder Veranstaltungen auch auf Artefakte gesetzt, wie das Gedächtnisspiel pexmory aus pex[eso] + [me]mory, bei dem durch das Hybridwort und das Spiel selbst die Sprachgrenze symbolisch und performativ aufgehoben und in einen Verflechtungsraum verwandelt wird.

\section{Fazit}

Zentral diskutiert wird in dem Beitrag die Relation von Sprache und Raum und damit auch von Sprache und Grenze, wobei dieser Raum einerseits horizontal in Bezug auf das Territorium, andererseits vertikal in Bezug auf die Macht und das Prestige in der Gesellschaft konzeptualisiert wird. Die Vorstellung der ,natürlichen' geografischen Grenzen sowie der Grenzen der Nationalstaaten als etwas objektiv Gegebenes wirkt zwar in der Sprachwissenschaft bis heute nach, die Reflexion der Rolle von Vorstellungen über die Sprachen und ihre Grenzen fand jedoch insbesondere in Bezug auf ihren Wandel bereits Eingang in die Variationslinguistik und wird sich wohl weiter vertiefen (vgl. Auer/Schmidt 2010). Das Letztere ist auch im Zusammenhang mit der sehr unterschiedlichen - interlingualen vs. intralingualen - Deutung von Sprachgrenzen deutlich geworden, wie dies etwa am Beispiel des Sorbischen konkretisiert wurde. Ähnliches trifft auch für die Reflexion von Sprachgrenzen zu, deren (vertikale) Verortung, Charakter und Ausprägung sich im Zuge der Entgrenzung und Informalisierung durch die neuen Medien grundsätzlich verändern, sowie für die Reflexion von Sprachgrenzen, die im Rahmen der Kontakt- und Interlinguistik sowie der Spracherwerbs- und Mehrspra- 
chigkeitsforschung untersucht werden. Angesichts der modernen Mehrsprachigkeit als Folge des Postkolonialismus, der Migration und der Verflechtung in urbanen Zentren sowie transund supranationalen Organisationsformen moderner Gesellschaft/en kann es eine trennscharfe Abgrenzung eines homogenen monolingualen Sprachraums, die der ethnonational getragene Sprachpurismus in der Sprachkultur durch die nationalen Philologien als Idealnorm gesetzt hat, auch kaum geben (vgl. auch Höfler/Klessmann in diesem Band). Vielmehr wird immer mehr die sprachliche Verflechtung sicht- und hörbar, der man etwa in den Herkunftssprachen, den Grenzorganisationen oder in den Sprachlandschaften und Interaktionen in den Grenzregionen begegnen kann und die bis in die hybriden Neuschöpfungen hineinreicht. So lässt sich die steigende Bedeutung von Cross Border Studies mit Fragen nach der Überschreitung von bestehenden interlingualen Grenzen sowie die steigende Bedeutung von linguistic mapping erwarten, das urbane Zentren als Mikrokosmen der Globalität adressiert, und zwar nicht nur mit Blick auf den Raum, in den diese eingeschrieben sind, sondern verstärkt auch auf die Sprachnutzer*innen und ihre Interaktion. Neu diskutiert und gedacht werden dürfte die Grenze auch in der heritage linguistics mit Blick auf die sprachliche Hybridisierung sowie in der Interaktionslinguistik mit Blick auf das Codeswitching bzw. Codemixing von Varietäten in den neuen Medien und von Sprachen in der Interaktion von Herkunftssprecher*innen. Unterbeleuchtet ist bisher die Frage der Grenze bei dem (intergenerationellen) Sprachwechsel (language shift), der in der Zeit geschieht, sich aber auch in der sprachlichen Hybridisierung manifestiert. Durch die Verknüpfung der an sich räumlichen Kategorie der Grenze mit der Zeit, die in der Interaktion im Codeswitching vorliegt und in der Interaktionslinguistik in Bezug darauf diskutiert wird, dürfte diese Fragestellung über die Sprachwissenschaft hinaus von Interesse sein.

\section{Weiterführende Literatur}

Auer, Peter (2005): Sprache, Grenze, Raum. In: Zeitschrift für Sprachwissenschaft 23, H. 2, S. 149-179.

Kimura, Goro Christoph (2018): Alternative interlingual strategies for crossing linguistic borders: Theoretical possibilities and their realization at the German-Polish border. In: Jańczak, Barbara Alicja (Hrsg.): Language Contact and Language Policies across Borders: Construction and Deconstruction of Transnational and Transcultural Spaces. Berlin: Logos, S. 73-88.

Marx, Christoph/Nekula, Marek (2015): Constructing a cross-border space through semiotic landscapes: A case study of a German-Czech organization. In: Laitinen, Mikko/Zabrodskaja, Anastassia (Hrsg): Dimensions of Sociolinguistic Landscapes in Europe: Materials and Methodological Solutions. Frankfurt/M.: Peter Lang, S. 149-168.

\section{Literaturverzeichnis}

Auer, Peter (1983): Zweisprachige Konversationen: Code-Switching und Transfer bei italienischen Migrantenkindern in Konstanz. Dissertation, Universität Konstanz.

Auer, Peter (2005): Sprache, Grenze, Raum. In: Zeitschrift für Sprachwissenschaft 23, H. 2, S. 149-179.

Auer, Peter (2013): Sprachliche Interaktion. Tübingen: Niemeyer.

Auer, Peter (2015): Auswirkungen der Staatsgrenze auf die Sprachsituation im Oberrheingebiet. In: Kehrein, Roland/Lameli, Alfred/ Rabanus, Stefan (Hrsg.): Regionale Variation des Deutschen. Berlin/Boston: De Gruyter, S. 323-348.

Auer, Peter/Schmidt, Jürgen Erich E. (Hrsg.) (2010): Language and Space. An International Handbook of Linguistic Variation. Theories and Methods. Berlin/New York: De Gruyter Mouton.

Bach, Adolf (1934/1969): Deutsche Mundartforschung. Heidelberg: Winter.

Backhaus, Peter (2007): Linguistic Landscapes: A Comparative Study of Urban Multilingualism in Tokyo. Clevedon: Multilingual Matters.

Bermel, Neil (2007): Linguistic Authority, Language Ideology, and Metaphor. The Czech Orthography Wars. Language, Power and Social Process. Berlin/New York: Mouton de Gruyter.

Bermel, Neil (2010): On the alleged Czech diglossia in the contemporary world. In: Slovo a slovesnost 71, H. 1, S. 5-30. 
Bermel, Neil (2014): Czech Diglossia: Dismantling or Dissolution? In: Arokay, Judit/Gvozdanovic, Jadranka/Miyajima, Darja (Hrsg.): Divided Languages? Diglossia, Translation and the Rise of Modernity in Japan, China, and the Slavic World. Cham: Springer International Publishing, S. 21-37.

Busch, Brigitta (2017): Mehrsprachigkeit, 2. Aufl. Wien: facultas.

Dietrich, Wolf (2019): Sprachgrenzen - Grenzen im Raum und in der individuellen Kompetenz. In: Kuhn, Barbara/Winter, Ursula (Hrsg.): Grenzen - Annäherungen an einen transdisziplinären Gegenstand. Würzburg: Königshausen \& Neumann, S. 233-256.

Ehlers, Klaas-Hinrich (2010): Der Wille zur Relevanz: Die Sprachforschung und ihre Förderung durch die DFG 1920-1970. Stuttgart: Franz Steiner Verlag.

Ferguson, Charles A. (1959): Diglossia. In: Word 15, S. 325-340.

Giger, Markus (2003): Standard und Nonstandard in der Tschechischen Republik und der deutschsprachigen Schweiz. In: Linguistica Brunensia A 51, S. 83-98.

Händler, Harald/Wiegand, Herbert Ernst (1989): Das Konzept der Isoglosse: methodische und terminologische Probleme. In: Besch, Werner/Knoop, Ulrich/Putschke, Wolfgang/Wiegand, Herbert Ernst (Hrsg.): Dialektologie. Ein Handbuch zur deutschen und allgemeinen Dialektforschung, Bd. 1. Berlin/New York: Walter de Gruyter, S. 501-527.

Haslinger, Peter (2010): Nation und Territorium im tschechischen politischen Diskurs 1880-1938. München: Oldenbourg.

Havránek, Bohuslav (1934): Nářečí česká. In: Hujer, Oldřich (Hrsg.): Československá vlastivěda, Bd. 3: Jazyk. Praha: Sfinx, S. 84-218.

Holly, Werner/Nekvapil, Jiř̌l/Scherm, Ilona/Tišerová, Pavla (2003): Unequal neighbours: Coping with asymmetries. In: Journal of Ethnic and Migration Studies 29, S. 819-834.

Hronek, Jiři/Sgall, Petr (1992): Čeština bez př́kras. Praha: H \& H.

Jańczak, Barbara Alicja (Hrsg.) (2018a): Language Contact and Language Policies across Borders: Construction and Deconstruction of Transnational and Transcultural Spaces. Berlin: Logos.

Jańczak, Barbara Alicja (2018b): Borderlands as spaces of transition: The communication of Polish Vendors in the German-Polish border region by the example of forms of address. In: Jańczak, Barbara Alicja (Hrsg.): Language Contact and Language Policies across Borders: Construction and Deconstruction of Transnational and Transcultural Spaces. Berlin: Logos, S. 89-103.

Janich, Nina (2007): Sprachplanung. In: Knapp, Karlfried (Hrsg.): Angewandte Linguistik. Tübingen/Basel: Francke, S. 537-558.

Kafka, Franz (1998): Briefe an Milena, erweiterte Neuausgabe, hg. von Jürgen Born und Michael Müller. Frankfurt/M.: Fischer.

Kimura, Goro Christoph (2018): Alternative interlingual strategies for crossing linguistic borders: Theoretical possibilities and their realization at the German-Polish border. In: Jańczak, Barbara Alicja (Hrsg.): Language Contact and Language Policies across Borders: Construction and Deconstruction of Transnational and Transcultural Spaces. Berlin: Logos, S. 73-88.

Kloss, Heinz (1966): Types of multilingual communities. In: Sociological Inquiry 36, S. 135-145.

König, Werner (1978): dtv-Atlas deutsche Sprache. München: Deutscher Taschenbuch Verlag.

Kremnitz, Georg (2005): Diglossie - Polyglossie / Diglossia - Polyglossia. In: Ammon, Ulrich/Dittmar, Norbert/Mattheier, Klaus J./Trudgill Peter (Hrsg.): Sociolinguistics / Soziolinguistik. Bd. 1.1, 2. Aufl. Berlin/New York: De Gruyter, S. 158-165.

Krempaská, Barbora (2019): Codeswitching in der Rede der Studierenden der Deutsch-Tschechischen Studien. Hausarbeit, Univerzita Karlova.

Kučera, Jaroslav (1999): Minderheit im Nationalstaat. Die Sprachenfrage in den tschechisch-deutschen Beziehungen 1918-1938. München: Oldenbourg.

Lamprecht, Arnošt/Šlosar, Dušan/Bauer, Jaroslav (1986): Historická mluvnice češtiny. Praha: SPN.

Lefebvre, Henri (2006): Die Produktion des Raumes. In: Dünne, Jörg J./Günzel, Stephan (Hrsg.): Raumtheorie. Grundlagentexte aus Philosophie und Kulturwissenschaften. Frankfurt/M.: Suhrkamp, S. 330343.

Marti, Roland (1993): Slovakisch und Čechisch vs. Čechoslovakisch, Serbokroatisch vs. Kroatisch und Serbisch. In: Gutschmidt, Karl/Keipert, Helmut/Rothe, Hans (Hrsg.): Slavistische Studien zum XI. internationalen Slavistenkongreß in Preßburg/Bratislava. Köln/Weimar/Wien: Böhlau, S. 289-315.

Marti, Roland (i.E.): Language Management from Above (and from Below), from Outside (and from Inside): The Case of Lower Sorbian. In: Nekula, Marek/Sherman, Tamah/Zawiszová, Halina (Hrsg.): Interests and Power in Language Management. Berlin: Peter Lang.

Marx, Christoph/Nekula, Marek (2014): Verständigung und Sprachmanagement in deutsch-tschechischen Grenzorganisationen. In: Engel, Nicolas/Göhlich, Michael/Höhne, Thomas/Klemm, Matthias/Kreatsch, Clemens/Marx, Nekula/Renn, Joachim (Hrsg.): Grenzen der Grenzüberschreitung: Zur „Übersetzungsleistung " deutsch-tschechischer Grenzorganisationen. Bielefeld: transcript, S. 45-122. 
Marx, Christoph/Nekula, Marek (2015): Constructing a cross-border space through semiotic landscapes: A case study of a German-Czech organization. In: Laitinen, Mikko/Zabrodskaja, Anastassia (Hrsg.): Dimensions of Sociolinguistic Landscapes in Europe: Materials and Methodological Solutions. Berlin: Lang, S. 149-168.

Matras, Yaron (2009): Language Contact. Cambridge: Cambridge University Press.

Muth, Sebastian (2014): Linguistic landscapes on the other side of the border: Signs, language, and the construction of cultural identity in Transnistria. In: International Journal of the Sociology of Language 227, S. 25-46.

Muysken, Pieter (2000): Bilingual Speech. A Typology of Code-Mixing. Cambridge: Cambridge University Press.

Nekula, Marek (2000): Franz Kafka und die tschechische Sprache. Zum Stil seiner tschechisch geschriebenen Texte. In: Stylistyka 9, S. 217-225.

Nekula, Marek (2002): Kommunikationsführung in deutsch-tschechischen Firmen. In: Möller, Joachim/Nekula, Marek (Hrsg.): Wirtschaft und Kommunikation. Beiträge zu deutsch-tschechischen Wirtschaftsbeziehungen. München: Iudicium, S. 65-83.

Nekula, Marek/Nekvapil, Jiř́/Šichová, Kateřina (2005a): Sprachen in deutsch-tschechischen, österreichisch-tschechischen und schweizerisch-tschechischen Unternehmen: Ein Beitrag zur Wirtschaftskommunikation in der Tschechischen Republik. In: Sociolinguistica 19, S. 128-143.

Nekula, Marek/Nekvapil, Jiři/Šichová, Kateřina (2005b): Sprachen in multinationalen Unternehmen auf dem Gebiet der Tschechischen Republik. München: FOROST-Arbeitspapier.

Nekula, Marek/Šichová, Kateřina (2004): Sprache als Faktor der wirtschaftlichen Integration. In: brücken - Germanistisches Jahrbuch 12, S. 317-335

Nekvapil, Jiří (2009): The integrative potential of Language Management Theory. In: Nekvapil, Jiří Sherman, Tamah (Hrsg.): Language Management in Contact Situations: Perspectives from three Continents. Frankfurt/M.: Peter Lang, S. 1-11.

Nekvapil, Jiří/Nekula, Marek (2006): On Language Management in Multinational Companies in the Czech Republic. In: Current Issues in Language Planning 7, H. 2-3, S. 307-327.

Nekvapil, Jiř́/Sherman, Tamah (2013): Language Ideologies and Linguistic Practices: The Case of Multinational Companies in Central Europe. In: Barát, Erszébet/Studer, Patrick/Nekvapil, Jiří (Hrsg.): Ideological Conceptualizations of Language. Discourses of Linguistic Diversity. Berlin: Peter Lang, S. 85-117.

Němec, Mirek (2009): Erziehung zum Staatsbürger? Deutsche Sekundarschulen in der Tschechoslowakei 1918-1938. Essen: Klartext.

Newerkla, Stefan Michael (1999): Intendierte und tatsächliche Sprachwirklichkeit in Böhmen: Diglossie im Schulwesen der böhmischen Kronländer 1740-1918. Wien: WUV.

Poplack, Shana/Sankoff, David (1988): Code-Switching. In: Ammon, Ulrich/Dittmar, Norbert/Mattheier, Klaus J. (Hrsg.): Sociolinguistics / Soziolinguistik, 2 Bd. Berlin/New York: Walter de Gruyter, S. 11741180.

Poplack, Shana (2004): Code-Switching. In: Ammon, Ulrich/Dittmar, Norbert/Mattheier, Klaus J./Trudgill, Peter (Hrsg.): Sociolinguistics: An International Handbook of the Science of Language and Society / Soziolinguistik: Ein internationales Handbuch zur Wissenschaft von Sprache und Gesellschaft, 3 Bd., 2., vollständig neu bearbeitete und erweiterte Aufl. Teilband 1. Berlin/New York: Walter de Gruyter, S. 589-596.

Raecke, Jochen (2002): Schlagbäume im sprachlichen Kontinuum: Wie Dialektgrenzen zu Sprachgrenzen werden (am Beispiel des kroatischen und slovenischen Kajkavischen). In: Zeitschrift für Slavische Philologie 61, H. 2, S. 337-364.

Riehl, Claudia Maria (2014): Sprachkontaktforschung: Eine Einführung. 3. erw. Aufl., Tübingen: Narr.

Schmidt, Johannes (1872): Die Verwandtschaftsverhältnisse der indogermanischen Sprachen. Weimar: Böhlau.

Schnabel, Michael (2006): Dialektspaltung im thüringisch-bayerischen Grenzgebiet am Beispiel des Ortspaars Sparnberg/Rudolphstein. Wie eine politische Grenze zur Sprachgrenze wurde. In: Zeitschrift für Dialektologie und Linguistik 73, H. 1, S. 30-54.

Sherman, Tamah/Nekvapil, Jiří (Hrsg.) (2018): English in Business and Commerce: Interactions and Policies. Boston/Berlin: Mouton de Gruyter.

Smits, Tom F. H. (2011): Strukturwandel in Grenzdialekten. Die Konsolidierung der niederländisch-deutschen Staatsgrenze als Dialektgrenze. Stuttgart: Franz Steiner.

Spolsky, Bernard/Cooper, Robert (1991): The Languages of Jerusalem. Oxford: Oxford University Press.

Stöhr, Ingrid (2010): Zweisprachigkeit in Böhmen: Deutsche Volksschulen und Gymnasien im Prag der Kafka-Zeit. Wien/Köln/Weimar: Böhlau. 
Švingrová, Simona (2010): Tschechisch oder Deutsch? Auf dem Weg von Konkurrenz zu Dominanz. Zum Einsatz von innerer und äußerer Amtssprache in der Arbeiter-Unfall-Versicherungs-Anstalt im Prag der Kafka-Zeit (1908-1922). Dissertation, Univerzita Karlova.

Vandermeeren, Sonja (1998): Fremdsprachen in europäischen Unternehmen. Waldsteinberg: Heidrun Popp.

Viereck, Wolfgang/Viereck, Karin/Ramisch, Heinrich (2002): dtv-Atlas Englische Sprache. München. Deutscher Taschenbuch Verlag.

Vuković, Petar (i.E.): Council for the Standard Croatian Language Norm: The Failure of „Hard Power“. In: Nekula, Marek/Sherman, Tamah/Zawiszová, Halina (Hrsg.): Interests and Power in Language Management. Berlin: Peter Lang.

Wenker, Georg (1886): Vortrag, veröffentlicht in Verhandlungen der 38. Versammlung deutscher Philologen und Schulmänner in Gießen vom 30.9. bis 3.10.1885. Leipzig: Teubner, S. 187-194.

Werlen, Iwar (2004): Zur Sprachsituation der Schweiz mit besonderer Berücksichtigung der Diglossie in der Deutschschweiz. In: Vereinigung für angewandte Linguistik in der Schweiz 79, S. 1-30.

Wolf, Michaela (2013): Die vielsprachige Seele Kakaniens: Übersetzen und Dolmetschen in der Habsburgermonarchie 1848 bis 1918. Köln/Weimar/Wien: Böhlau, S. 87-193.

Woolard, Kathryn A. (1998): Introduction: Language ideology as a field of inquiry. In: Schieffelin, Bambi B./Woolard, Kathryn A. et al. (Hrsg.): Language Ideologies: Practice and Theory. Oxford/New York: Oxford University Press, S. 3-47. 